\title{
Anchoring of a Nematic Liquid Crystal on a Wettability Gradient
}

\author{
Andrew D. Price and Daniel K. Schwartz \\ Department of Chemical and Biological Engineering, University of Colorado, Boulder, \\ Colorado 80309
}

\section{SUPPORTING INFORMATION}

Additional motivation for understanding the effects of surface chemistry on nematic anchoring comes from recent work where one anchoring surface is provided by a monolayer of adsorbed amphiphilic molecules at the aqueous/nematic interface. ${ }^{1}$ Applications for the configuration are proposed where the nematic anchoring transition provides a macroscopic optical signal in response to subtle structural changes within the interfacial monolayer; e.g. in response to a molecular recognition event. This response, however, is complicated and certainly not binary. Fig. 1, for example, shows the dynamic response of a HAN cell as surfactant gradually absorbs on the nematic/aqueous interface. The temporal progression of effective birefringence colors suggests that the nematic anchoring varies continuously with surface concentration in a way that is not completely understood. Unfortunately, it is difficult to independently study the properties of the surface monolayer in this configuration. This provided the motivation to develop a related system where the properties of an aliphatic surface could be varied continuously and characterized directly, to enable correlation of surface chemistry and nematic anchoring.

\section{Surfactant Adsorption Experiments}

The nematic LC was contained within 20 micron thick copper grids (Electron Microscopy Sciences) placed on OTE-SAM-treated glass. 5CB was deposited in the grid using a $25 \mu \mathrm{l}$ 
capillary tube, and then immersed in a solution of $0.5 \mathrm{mM}$ dodecyltrimethylammonium bromide (DTAB) in $1 \mathrm{X}$ phosphate buffered saline, $\mathrm{pH} 7.4$ at room temperature.

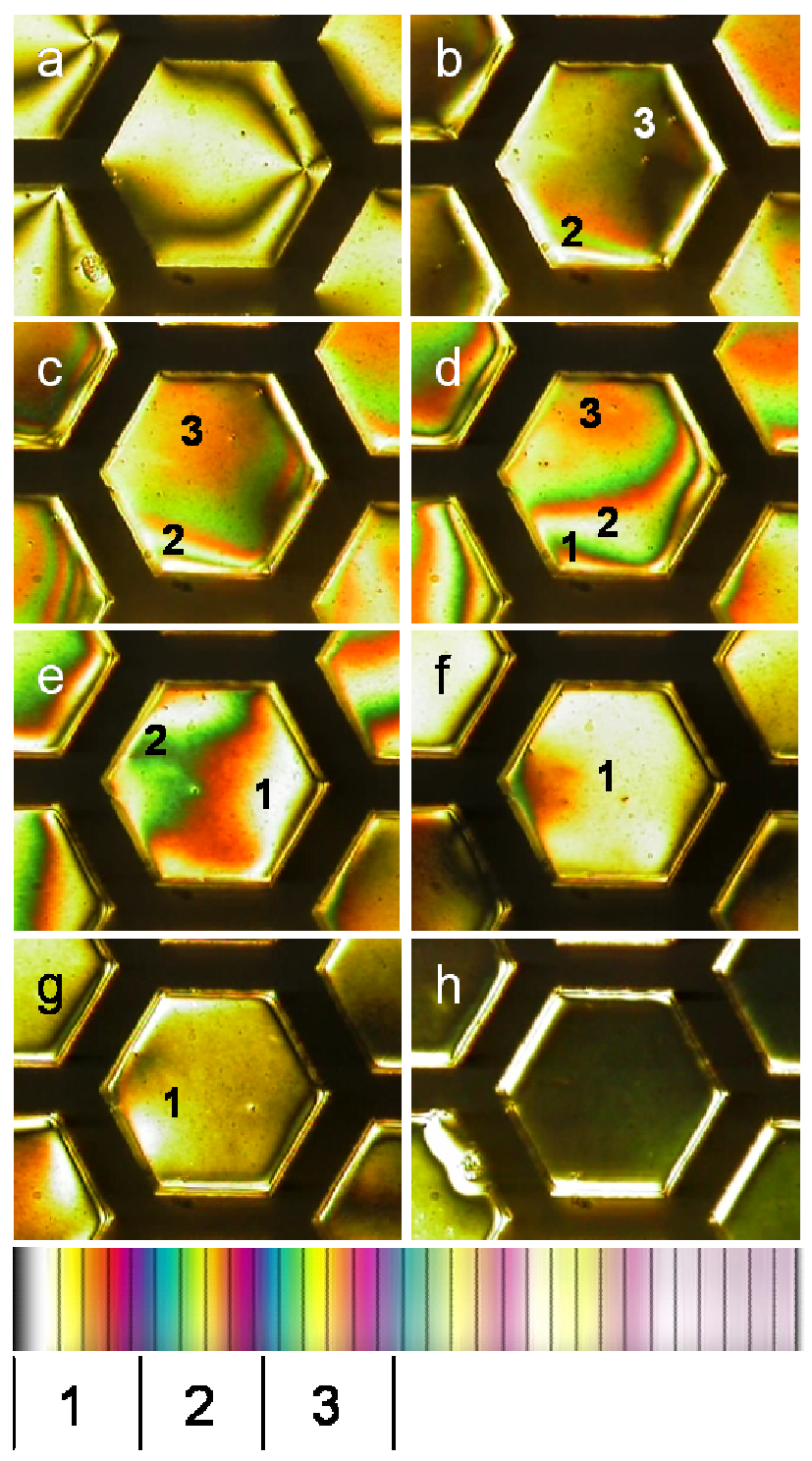

Figure 1. The adsorption of $0.5 \mathrm{mM}$ DTAB at the aqueous/LC interface (a-h) created effective birefringence patterns that progressed from third order to first order birefringence colors as the surface concentration of surfactant increased. The order of the transition is noted in the images. In image $h$ the effective birefringence vanished as the DTAB monolayer density was great enough to induce homeotropic anchoring.

\section{Surfactant Adsorption}

Similar to a surface varying in its surface wettability, the adsorption of amphiphilic molecules at an aqueous/5CB interface creates a dynamic interface that also permits the observation of effective birefringence patterns due to anchoring by an adsorbing monolayer. Previous work by 
Brake and $\mathrm{Abbott}^{1}$ showed that $5 \mathrm{CB}$ adopts a planar alignment at a clean aqueous/LC interface, but that the adsorption of surfactant at the aqueous/5CB interface induced a transition to a uniformly homeotropic alignment. Dynamic observations during surfactant adsorption, however (see Fig. 1), suggest that there is intermediate behavior as the monolayer becomes increasingly densely packed. In other words, intermediate values of birefringence are observed, in analogy with the transition region on gradient surfaces. Figure 1 shows a time progression of a $20 \pm 2 \mu \mathrm{m}$ thick 5CB layer immersed in $0.5 \mathrm{mM}$ DTAB. Initially, Fig. 1a, there was a large pretilt (approximately planar alignment) at the aqueous/5CB interface with only a brush structure present in regions where the azimuthal LC angle is parallel to the polarizer or analyzer. As DTAB adsorbed, the effective birefringence decreased as the tilt angle at the aqueous/LC interface decreased. The transition from third order to first order birefringence presumably represented regions of increasing packing density of DTAB at the interface. These regions are labeled in the figure. The decrease in birefringence order proceeded across the grid hole as "color fronts" until the effective birefringence disappeared altogether indicating a uniform homeotropic alignment of the liquid crystal layer. It should be noted that a continuous decrease in effective birefringence was expected for DTAB since it formed a fairly uniform Gibbs monolayer at the aqueous/5CB interface exhibiting only a single phase. Other surfactants, such as palmitic acid, were observed to cause distinct regions of zero birefringence. ${ }^{2}$ This is presumed to be due to coexisting phases in the adsorbed monolayer.

\section{REFERENCES}

(1) Brake, J. M.; Abbott, N. L. Langmuir 2002, 18, 6101-6109.

(2) Brake, J. M.; Daschner, M. K.; Abbott, N. L. Langmuir 2005, 21, 2218-2228. 